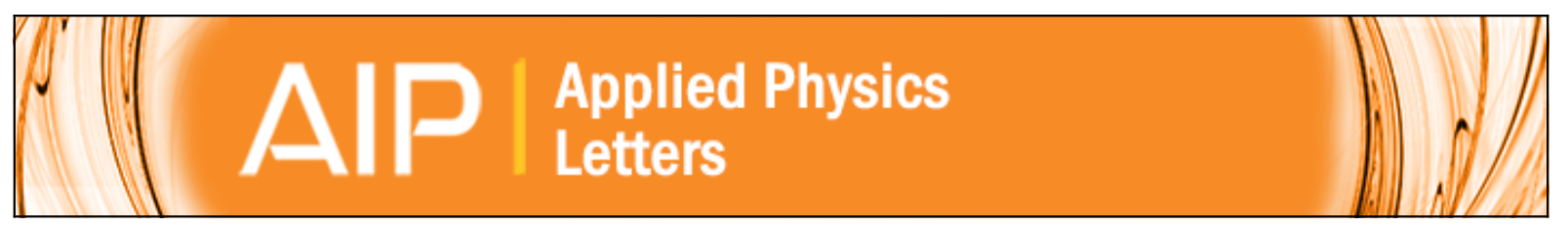

\title{
Uncorrelated multiple conductive filament nucleation and rupture in ultra-thin high-k dielectric based resistive random access memory
}

Xing Wu, Kun Li, Nagarajan Raghavan, Michel Bosman, Qing-Xiao Wang, Dongkyu Cha, Xi-Xiang Zhang, and Kin-Leong Pey

Citation: Applied Physics Letters 99, 093502 (2011); doi: 10.1063/1.3624597

View online: http://dx.doi.org/10.1063/1.3624597

View Table of Contents: http://scitation.aip.org/content/aip/journal/apl/99/9?ver=pdfcov

Published by the AIP Publishing

\section{Articles you may be interested in}

Multi-scale quantum point contact model for filamentary conduction in resistive random access memories devices J. Appl. Phys. 115, 244507 (2014); 10.1063/1.4885419

Total ionizing dose effect of $\mathrm{y}$-ray radiation on the switching characteristics and filament stability of HfOx resistive random access memory

Appl. Phys. Lett. 104, 183507 (2014); 10.1063/1.4875748

Low power W:AlOx/WOx bilayer resistive switching structure based on conductive filament formation and rupture mechanism

Appl. Phys. Lett. 102, 173503 (2013); 10.1063/1.4803462

Semiconducting-like filament formation in $\mathrm{TiN} / \mathrm{HfO} 2 / \mathrm{TiN}$ resistive switching random access memories

Appl. Phys. Lett. 100, 142102 (2012); 10.1063/1.3696672

Chemical insight into origin of forming-free resistive random-access memory devices

Appl. Phys. Lett. 99, 133504 (2011); 10.1063/1.3645623

You don't

still use this

cell phone

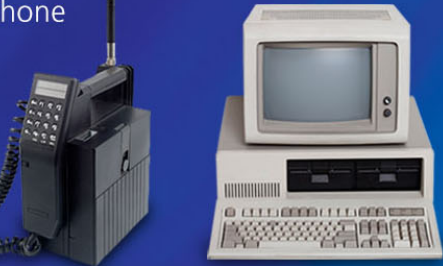

Why are you still using an AFM designed in the $80^{\prime}$ 's?
It is time to upgrade your AFM

Minimum \$20,000 trade-in discount for purchases before August 31st

Asylum Research is today's technology leader in AFM 


\title{
Uncorrelated multiple conductive filament nucleation and rupture in ultra-thin high- $\kappa$ dielectric based resistive random access memory
}

\author{
Xing Wu, ${ }^{1}$ Kun Li, ${ }^{2}$ Nagarajan Raghavan, ${ }^{1}$ Michel Bosman, ${ }^{3}$ Qing-Xiao Wang, ${ }^{2}$ \\ Dongkyu Cha, ${ }^{2}$ Xi-Xiang Zhang, ${ }^{2}$ and Kin-Leong Pey ${ }^{1,4, a)}$ \\ ${ }^{1}$ Division of Microelectronics, School of Electrical and Electronic Engineering, Nanyang Technological \\ University, Nanyang Avenue, Singapore 639798, Singapore \\ ${ }^{2}$ Imaging and Characterization Core lab, King Abdullah University of Science and Technology, Thuwal, \\ Kingdom of Saudi Arabia, 23955-6900, Kingdom of Saudi Arabia \\ ${ }^{3}$ Institute of Materials Research and Engineering, A*STAR (Agency for Science, Technology and Research), \\ 3 Research Link, Singapore 117602, Singapore \\ ${ }^{4}$ Engineering Product Development Pillar, Singapore University of Technology and Design, 20 Dover Drive, \\ Singapore 138682, Singapore
}

(Received 16 May 2011; accepted 21 July 2011; published online 29 August 2011)

\begin{abstract}
Resistive switching in transition metal oxides could form the basis for next-generation non-volatile memory (NVM). It has been reported that the current in the high-conductivity state of several technologically relevant oxide materials flows through localized filaments, but these filaments have been characterized only individually, limiting our understanding of the possibility of multiple conductive filaments nucleation and rupture and the correlation kinetics of their evolution. In this study, direct visualization of uncorrelated multiple conductive filaments in ultra-thin $\mathrm{HfO}_{2}$-based high- $\kappa$ dielectric resistive random access memory (RRAM) device has been achieved by high-resolution transmission electron microscopy (HRTEM), along with electron energy loss spectroscopy (EELS), for nanoscale chemical analysis. The locations of these multiple filaments are found to be spatially uncorrelated. The evolution of these microstructural changes and chemical properties of these filaments will provide a fundamental understanding of the switching mechanism for RRAM in thin oxide films and pave way for the investigation into improving the stability and scalability of switching memory devices. (C) 2011 American Institute of Physics. [doi:10.1063/1.3624597]
\end{abstract}

Resistive random access memory (RRAM) is one of the most promising candidates for the next generation of non-volatile memory (NVM) given its advantages in terms of fast writing/erasing times $(<100 \mathrm{~ns})$, long retention lifetime ( $>10 \mathrm{yr}$ ), low power consumption, and complementary metal-oxide-semiconductor (CMOS) technology compatibility. ${ }^{1-5}$ In addition, RRAM is also suitable for the three-dimension stacking of memory layers, which can prompt the realization of very high-density memory. ${ }^{6}$

The operational mechanism of RRAM is based on a reversible breakdown of an insulating dielectric. It is a simple two-terminal device in which the resistance of the device can be reversibly and repeatedly switched between a high resistance state (HRS) and a low resistance state (LRS) for different applied voltages. From a microscopic point of view, ${ }^{3}$ the resistive switching mechanisms are usually classified into electrochemical metallization mechanism, ${ }^{7,8}$ valence change mechanism, ${ }^{9-14}$ and the fuse-antifuse mechanism. ${ }^{15-17}$ Both bipolar and unipolar switching behaviors have been reported in $\mathrm{HfO}_{2}$ based dielectric stacks. ${ }^{18}$ Several other dielectric films also show similar non-polar switching behaviours. ${ }^{19,20}$ For all the mechanisms proposed, the switching behavior is predominantly due to the formation and rupture of a localized nanoscale conductive filament. However, the previous studies do not consider the possibility and implications of multiple conductive filamentation events. In this study, in-depth electrical characterization was carried out showing that the conduc-

\footnotetext{
${ }^{\text {a) }}$ Author to whom correspondence should be addressed. Electronic mail: peykinleong@sutd.edu.sg. Tel.: 65-67906371.
}

tive filaments are spatially and temporally uncorrelated during formation. Moreover, multiple conductive filaments undergo rupture individually.

The devices tested were conventional small area n-type metal-oxide-semiconductor (MOS) transistors with gate length of $L=350 \mathrm{~nm}$ and gate width $W=150 \mathrm{~nm}$, consisting of NiSi as the metal gate (top electrode, TE), a sandwiched dual-layer oxide of $\mathrm{HfO}_{2}(25 \AA)-\mathrm{SiO}_{2}(12 \AA)$, and $\mathrm{Si}$ as the bottom electrode (BE). The advantage of using $\mathrm{p}^{+}-\mathrm{Si}$ as $\mathrm{BE}$ is that the interface between the oxide and $\mathrm{Si}$ is very smooth, as compared to Pt based stacks which tend to show a rough interface. ${ }^{21}$ Figure 1 shows the SET and RESET $I-V$ trends achieved using a bipolar switching mode. Breakdown, in this case is the SET during the switching, is defined as the instant of a more than two times instantaneous increase in the current level during the voltage ramping. The first breakdown/ formation of the conductive filament occurred at $4.3 \mathrm{~V}$ (see the inset of Fig. 1), followed by another abrupt increase in the current at $4.8 \mathrm{~V}$, when the current reached the compliance setting of $I_{\text {com }}=1 \mathrm{~mA}$. The relative breakdown location $\left(\mathrm{S}=I_{d} /\left(I_{d}+I_{S}\right)\right)$ along the channel from the source $(\mathrm{S}=0)$ to drain $(\mathrm{S}=1)$ end is measured in accumulation/inversion mode using the technique proposed by Degraeve et al., ${ }^{22}$ Using this method, we found that the first conductive path formed at $\mathrm{S}=0.06$, which was near the source. After the second SET, we found $I_{s} \sim I_{d}$, indicating the effective conductive filaments location is in the center of the channel. This is verified to be true later by the TEM micrograph which shows physical evidence of conductive filaments at both edges of the transistor, implying the contribution of $I_{s}$ and $I_{d}$ is likely 


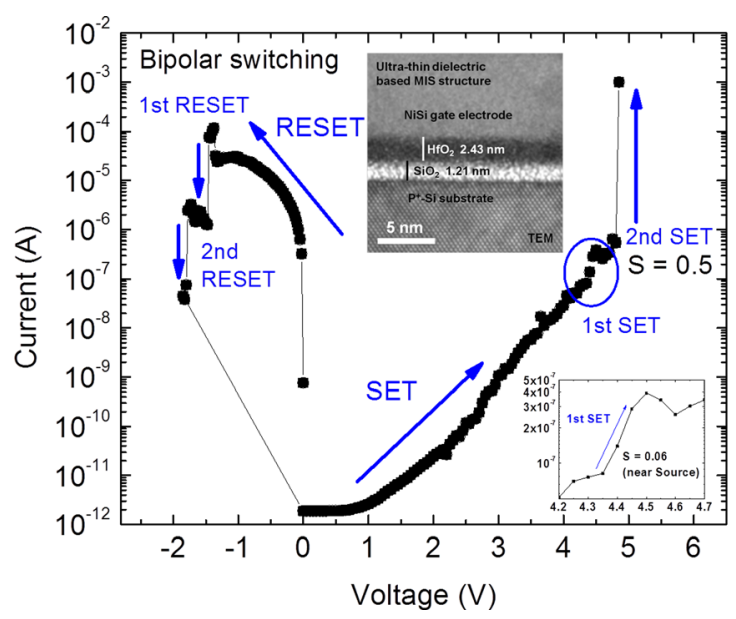

FIG. 1. (Color online) SET and RESET in a bipolar resistive switching device. Top inset shows the cross-sectional TEM micrograph of the ultra-thin dielectric based $\mathrm{NiSi} / \mathrm{HfO}_{2} / \mathrm{SiO}_{2} / \mathrm{p}^{+}-\mathrm{Si}$ MIS structure. The electrical data shows the bipolar $I-V$ switching trends where the compliance current was capped to $1 \mathrm{~mA}$. Two discrete increases in the current (SET) that accompany the formation of two individual conductive paths have been identified. The first SET occurred at voltage $+4.2 \mathrm{~V}$ with $\mathrm{S}=0.06$, which indicates the first conductive path was near the source. The bottom inset shows the details of the first SET. The second SET occurred at voltage $+4.8 \mathrm{~V} . I_{s} \sim I_{d}$, so the $\mathrm{S}$ value was 0.5 , implying the effective conductive paths location is in the center of the channel. The second conductive path might be at the drain edge of the transistor since the contribution of $I_{s}$ and $I_{d}$ were roughly the same. Two discrete RESETs have been observed during the negative voltage sweep. The current first abruptly decreased from $80 \mu \mathrm{A}$ to $1 \mu \mathrm{A}$ at $-1.3 \mathrm{~V}$, followed by a second current decreased from about $2 \mu \mathrm{A}$ to $80 \mathrm{nA}$ at $-1.7 \mathrm{~V}$. Both the switching events are of about two orders in magnitude change in the gate current.

to be roughly the same. During the negative voltage sweep from $0 \mathrm{~V}$ to $-2 \mathrm{~V}$, the gate leakage current first decreased at $-1.3 \mathrm{~V}$, followed by a second abrupt current decrease at $-1.7 \mathrm{~V}$. The range of voltages for bipolar switching here is from $-2 \mathrm{~V}$ to $+5 \mathrm{~V}$ with an $\mathrm{ON} / \mathrm{OFF}$ ratio $=1000$.

Our previous study shows that the conductive filament consists of oxygen vacancy clusters surrounding the Ni-rich metal nano-filament ( $2 \mathrm{~nm}$ in diameter). ${ }^{18}$ Figure 2 shows the post-RESET high angle annular dark field (HAADF)

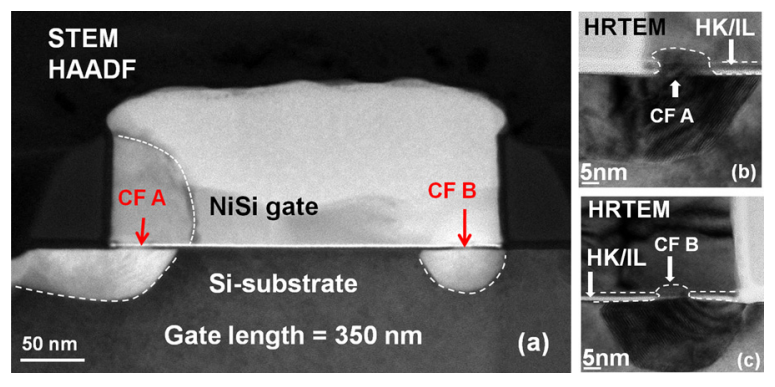

FIG. 2. (Color online) (a) STEM-HAADF micrographs of the MIS device in Fig. 1 after RESET showing two ruptured CFs: CF A and CF B. The bright contrast of the region in the $\mathrm{Si}$-substrate beneath $\mathrm{CF} \mathrm{A}$ is the lateral diffusion of Ni-based silicide compounds from the source, which is termed as DBIM (Refs. 22-24). DBIM can also be the vertical diffusion of NiSi from TE (anode) into the broken-down path in the dielectric. DBIM from both lateral and vertical directions could be the physical defects responsible for CF A and CF B, forming a sub-capacitor-like MIM structure. (b) and (c) High resolution TEM micrographs of $\mathrm{CF} A$ and $\mathrm{CF} B$, respectively. Here, the term $\mathrm{HK}$ represents the high- $\kappa$ dielectric of $\mathrm{HfO}_{2}$, while the term IL represents the interfacial layer of $\mathrm{SiO}_{2}$. The diameter of the ruptured conductive filament is around $5-10 \mathrm{~nm}$. scanning transmission electron microscope (STEM) micrograph of the device tested in Fig. 1. It can be clearly seen that there are two uncorrelated conductive paths at the source and drain ends labeled as "conductive filament (CF) A" and "CF B." The bright contrast region in the Si-substrate indicates that there is a presence of heavy metal atom, which is confirmed to be Ni-based materials by electron energy loss spectroscopy (EELS). Our previous study shows that the vertical migration of metal atoms from the transistor gate electrode and lateral diffusion of Ni-based silicide compounds from the source and drain into the broken-down high- $\kappa$ gate dielectric is the physical defect responsible for the highly conductive breakdown path, which is termed as the dielectric-breakdown-induced metal migration (DBIM). ${ }^{23-25}$ By monitoring the $I_{s}-I_{d}$ during SET process, the first breakdown spot occurred at the source (CF A) with DBIM, creating an MIM (metal insulator metal)-like sub-structure. The Ni in the dielectric could be from the gate electrode or DBIM formed in the substrate. Before CF B forms, CF A continues to degrade upon stressing, inducing more severe DBIM. Note that the Ni metal filament in CF B migrated from the gate electrode (anode) and the drain NiSi contact.

To further understand the chemical nature of the ruptured filament, EELS measurements were performed using a monochromated probe $\mathrm{C}_{\mathrm{S}}$ corrected FEI TITAN CUBED system, under high-tension voltage of $200 \mathrm{kV}$. The probe size was set to $0.9 \AA$ in diameter to obtain sub-angstrom level resolution, and the point to point distance is $1.3 \AA$ in the EELS line scan. The EELS spectra were collected from the central region of the ruptured filament, a region next to the ruptured filament and locations free of any filament present. Figure 3 shows the $\mathrm{Ni}$ and $\mathrm{O}$ EELS line profiles acquired from the two conductive filaments shown in Fig. 1 after RESET. The corresponding STEM HAADF micrographs shown in Figs. 3(b) and 3(d) illustrate the vertical EELS line scan locations. In CF A, the oxygen atoms signal distribution in the ruptured filament is different from the non-filamentary location and the region absence of the filament. At the same time, Ni signal is present in the dielectric of the ruptured filament. For the ruptured filament EELS signal, the lowest Ni signal intensity point correlates well with the highest $\mathrm{O}$ signal, which might be the localized location of discontinuity upon RESET. In CF B, the localized rupturing point of the conductive filament is in the middle. Metal filament nucleation and rupture are feasible at both CF A and B because of the formation of MIM-like stack due to the formation of DBIM at the Si substrate. Since Ni remains in the ruptured filament, oxidation of $\mathrm{Ni} \rightarrow \mathrm{NiO}_{\mathrm{x}}$ (insulator) may be a plausible explanation for the RESET. The chemical and structural change after conductive filament formation and rupture in this device might be the following: $\mathrm{Ni}$ metal filamentation, oxygen ion drift and storage in the metal electrode, and DBIM. All of these effects are favorable for the formation of conductive filament. The rupture of the conductive filament in bipolar reset can be realized by a combined effect of Joule heating and oxygen re-passivation from the gate electrode. Note that the role played by $\mathrm{O}^{2-}$ ions alone in the bipolar switching process is more obvious at very low switching compliance SET transitions. ${ }^{26}$

Our physical analysis results clearly show that the nucleation and rupture of conducting nanoscale metallic filaments 

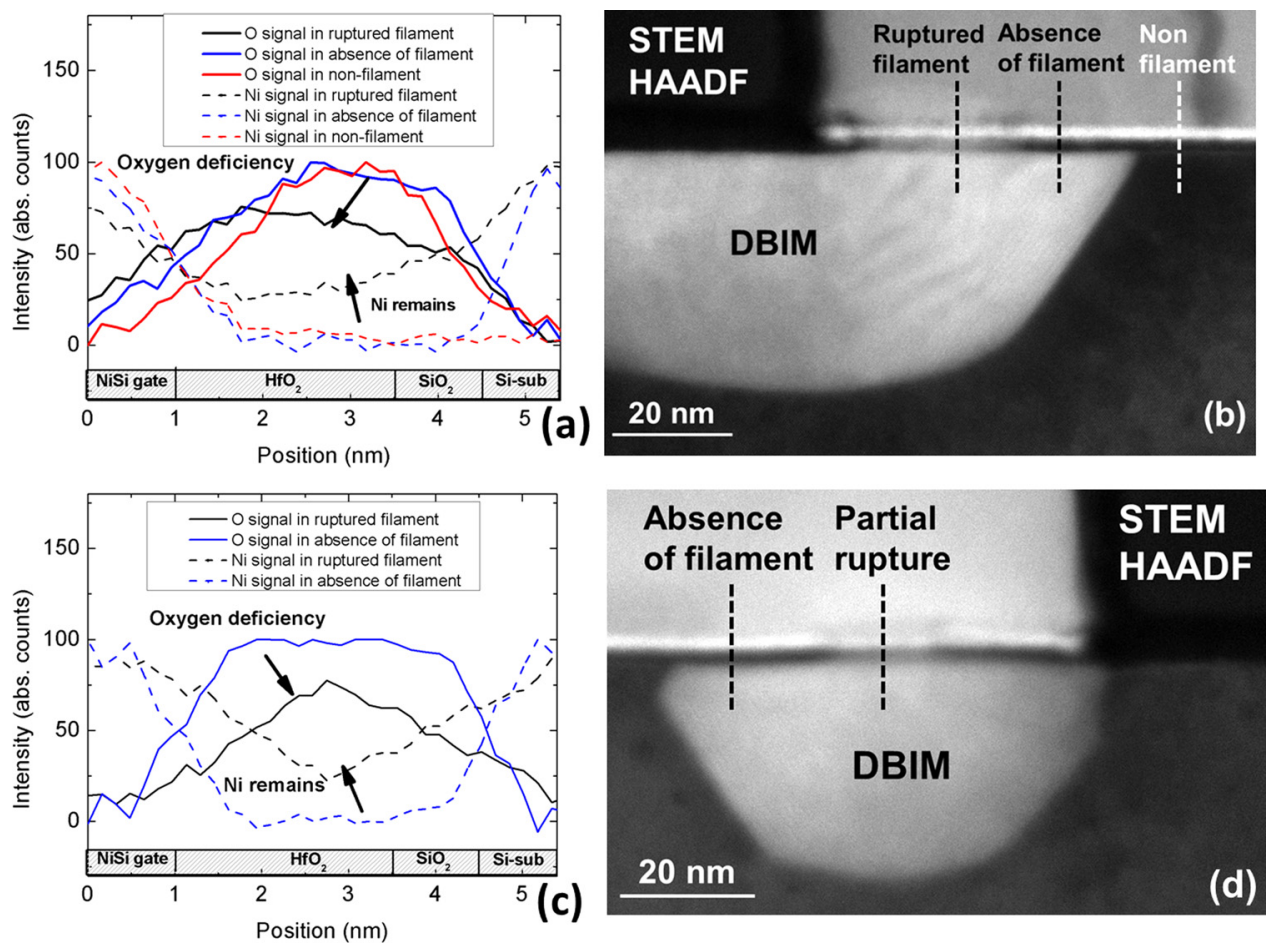

FIG. 3. (Color online) (a) Ni (dashed line) and $\mathrm{O}$ (solid line) EELS signal profiles for the line scans across the NiSi TE, the dual-layer dielectric of $\mathrm{HfO}_{2}$ and $\mathrm{SiO}_{2}$, and the $\mathrm{Si}$ substrate at three different locations near the $\mathrm{CF}$ A region. The horizontal line is the position along the stack, while the vertical line is the intensity of each chemical element signal. The intensity values are normalized for comparison. The three line scan locations are shown in (b): a ruptured filament (black line), a region absent of the ruptured filament above DBIM (blue line), and a non-filament spot (red line). At the ruptured filament location, the $\mathrm{O}$ intensity is lower than the absence of filament region, while Ni diffuses into the dielectrics. (c) and (d) Ni (dashed line) and $\mathrm{O}$ (solid line) EELS line profiles of CF B acquired at two locations indicated in the inset STEM micrograph. Similar $\mathrm{Ni}$ and $\mathrm{O}$ distribution trends as in $\mathrm{CF} \mathrm{A}$ have been observed. are responsible for the resistive switching phenomenon, while oxygen ion drift in the vicinity of the conductive filament also plays a role in the bipolar mode switching process. We demonstrate that multiple conductive filaments can be individually SET and RESET and are spatially uncorrelated. This means that further scalability of RRAM devices depends on the size of the conductive filaments as well as the extent of rupture/ dissolution of the conductive filament after each RESET, which has a strong impact on the endurance of the device.

$\mathrm{X}$. Wu would like to thank Gang Zhang from Peking University, China and Ping Bai from Institute of High Performance Computing (IHPC), A*STAR, Singapore for their technical discussions. $\mathrm{X}$. Wu gratefully acknowledges the scholarship support provided by the Ministry of Education (MOE), Singapore (Grant No. T206B1205). This research work is funded by the Singapore University of Technology and Design (SUTD) Research Grant No. SRG ASPE 2010004.

${ }^{1}$ G. W. Burr, B. N. Kurdi, J. C. Scott, C. H. Lam, K. Gopalakrishnan, and R. S. Shenoy, IBM J. Res. Dev. 52(4-5), 449 (2008).

${ }^{2}$ Q. Liu, S. Long, H. Lv, W. Wang, J. Niu, Z. Huo, J. Chen, and M. Liu, ACS Nano 4(10), 6162 (2010).

${ }^{3}$ R. Waser, R. Dittmann, C. Staikov, and K. Szot, Adv. Mater. 21(25-26), 2632 (2009).

${ }^{4}$ A. Sawa, Mater. Today 11(6), 28 (2008).

${ }^{5}$ G. I. Meijer, Science 319(5870), 1625 (2008).

${ }^{6}$ C. Kügeler, M. Meier, R. Rosezin, S. Gilles, and R. Waser, Solid-State Electron. 53(12), 1287 (2009).

${ }^{7}$ Z. Xu, Y. Bando, W. Wang, X. Bai, and D. Golberg, ACS Nano 4(5), 2515 (2010).

${ }^{8}$ M. N. Kozicki, M. Park, and M. Mitkova, IEEE Trans. Nanotechnol. 4(3), 331 (2005).
${ }^{9}$ D. H. Kwon, K. M. Kim, J. H. Jang, J. M. Jeon, M. H. Lee, G. H. Kim, X. S. Li, G. S. Park, B. Lee, S. Han, M. Kim, and C. S. Hwang, Nat. Nanotechnol. 5(2), 148 (2010).

${ }^{10}$ H. Y. Lee, Y. S. Chen, P. S. Chen, T. Y. Wu, F. Chen, C. C. Wang, P. J. Tzeng, M. J. Tsai, and C. Lien, IEEE Electron Device Lett. 31(1), 44 (2010).

${ }^{11}$ M. J. Lee, S. Han, S. H. Jeon, B. H. Park, B. S. Kang, S. E. Ahn, K. H. Kim, C. B. Lee, C. J. Kim, I. K. Yoo, D. H. Seo, X. S. Li, J. B. Park, J. H. Lee, and Y. Park, Nano Lett. 9(4), 1476 (2009).

${ }^{12}$ Y. B. Nian, J. Strozier, N. J. Wu, X. Chen, and A. Ignatiev, Phys. Rev. Lett. 98(14), 146403 (2007).

${ }^{13}$ J. Y. Son, C. H. Kim, J. H. Cho, Y. H. Shin, and H. M. Jang, ACS Nano 4(6), 3288 (2010).

${ }^{14}$ N. Raghavan, K. L. Pey, X. Wu, W. H. Liu, X. Li, M. Bosman, and T. Kauerauf, IEEE Electron Device Lett. 32(3), 252 (2011).

${ }^{15}$ G. A. Vorob'ev and N. S. Nesmelov, Russ. Phys. J. 22(1), 70 (1979).

${ }^{16}$ U. Russo, D. Ielmini, C. Cagli, and A. L. Lacaita, IEEE Trans. Electron Devices 56(2), 193 (2009).

${ }^{17}$ U. Russo, D. Ielmini, C. Cagli, and A. L. Lacaita, IEEE Trans. Electron Devices 56(2), 186 (2009).

${ }^{18}$ X. Li, W. H. Liu, N. Raghavan, M. Bosman, and K. L. Pey, Appl. Phys. Lett. 97, 202904 (2010).

${ }^{19}$ Y. C. Yang, F. Pan, Q. Liu, M. Liu, and F. Zeng, Nano Lett. 9(4), 1636 (2009).

${ }^{20}$ D. S. Jeong, H. Schroeder, and R. Waser, Electrochem. Solid-State Lett. 10(8), G51 (2007).

${ }^{21}$ Z. Fang, H. Y. Yu, W. J. Liu, Z. R. Wang, X. A. Tran, B. Gao, and J. F. Kang, IEEE Electron Device Lett. 31(5), 476 (2010).

${ }^{22}$ R. Degraeve, B. Kaczer, A. De Keersgieter, and G. Groeseneken, IEEE Trans. Device Mater. Reliab. 1(3), 163 (2001).

${ }^{23}$ X. Li, K. L. Pey, M. Bosman, W. H. Liu, and T. Kauerauf, Appl. Phys. Lett. 96(2), 022903 (2010).

${ }^{24}$ R. Ranjan, K. L. Pey, C. H. Tung, D. S. Ang, L. J. Tang, T. Kauerauf, R. Degraeve, G. Groeseneken, S. De Gendt, and L. K. Bera, Appl. Phys. Lett. 88(12), 122907 (2006).

${ }^{25}$ L. J. Tang, K. L. Pey, C. H. Tung, M. K. Radhakrishnan, and W. H. Lin, IEEE Trans. Device Mater. Reliab. 4(1), 38 (2004).

${ }^{26}$ N. Raghavan, K. L. Pey, X. Li, W. H. Liu, X. Wu, M. Bosman, and T. Kauerauf, IEEE Electron Device Lett. 32(6), 252 (2011). 